\title{
The Liverpool Telescope Spectrograph: FRODOSpec
}

\author{
L. Morales-Rueda ${ }^{1}$, D. Carter ${ }^{2}$, I. A. Steele ${ }^{2}$, P. A. Charles ${ }^{1}$, S. Worswick ${ }^{3}$ \\ 1. University of Southampton, UK, 2. Liverpool John Moores University, UK, 3. Observatory Optics, 1 Betoni Vale, UK
}

Received date will be inserted by the editor; accepted date will be inserted by the editor

\section{The Liverpool Telescope \& FRODOSpec}

The Liverpool Telescope (LT) is a $2 \mathrm{~m}$ fully robotic Cassegrain telescope sited at the Observatorio del Roque de los Muchachos in La Palma. Three main instruments are being built for use at this telescope: an optical CCD camera, a near infra-red camera and an integral field unit, the Fibre-fed RObotic Dual-beam Optical Spectrograph (FRODOSpec).

FRODOSpec's front end consists of a lenslet array with each lenslet fed to an optical fibre. The output fibres are lined up in a pseudo-slit and passed through a dichroic beamsplitter, allowing us to separate the blue and red regions of the spectrum. The optics that follow are then optimised for each wavelength region thereby maximising the total throughput. Each beam or arm contains two different dispersing elements giving 2 resolutions in the red and two in the blue. The final result is an intermediate resolution spectrograph $(\mathrm{R} \sim 4000)$ that covers the full optical wavelength range from 3800 to $9500 \AA$, with a higher resolution option $(\mathrm{R} \sim 8000)$ covering two wavelength ranges, $3800-5600$ and $5600-7400 \AA$.

FRODOSpec, with an integral field unit field of view of $11 \times 11$ arcsecs, has been designed mainly to study point sources. The robotic nature of the telescope plus spectrograph will allow us to monitor objects over a wide range of temporal baselines. FRODOSpec will be able to achieve a time resolution between 5 and $10 \mathrm{~s}$. We will also make use of the target of opportunity advantage that a robotic telescope can give, e.g. this will allow us to study dwarf novae in their outburst state which is the step needed for understanding the viscous evolution of their accretion discs and thereby constrain the nature of that viscosity. The third advantage of a robotic spectrograph is to permit queue scheduling, whereby observations for a particular project will take place when the conditions are optimal, e.g. given orbital phase, seeing.

Time-lapse spectroscopy by FRODOSpec will allow us to use Doppler Tomography to perform indirect imaging of interacting binaries on the microarcsecond scale. We are also considering the addition of polarimetric capabilities to the spectrograph in the future. This would allow observers to perform Stokes Imaging and Zeeman Doppler Tomography. For

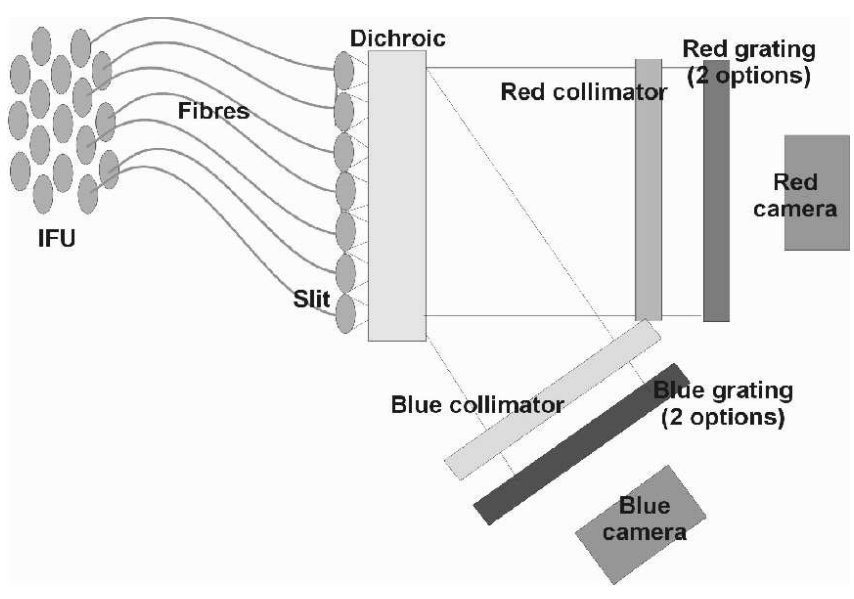

Fig. 1. Schematic layout for FRODOSpec.

1-dimensional indirect imaging techniques like Eclipse Mapping and Physical Parameter Eclipse Mapping, observers can obtain colour photometry with the CCD camera.

\section{Useful facts}

The breakdown of the available time on the LT is as follows: $70 \%$ for the United Kingdom (of which $30 \%$ is for John Moores University), 20\% for Spanish astronomers, 5\% for international applicants and the remaining $5 \%$ for education and public understanding of science in the UK. Observing time will be allocated by PATT in the UK, CAT in Spain and CCI for the international time. Successful applicants will be given fully reduced data. The observing time is assigned in hours and the switch between imaging and spectroscopy is effected via the positioning of a deployable tertiary mirror. The data will be public and stored in an archive one year after the observations for a given programme are completed.

FRODOSpec is being developed jointly by Southampton and Liverpool John Moores Universities. For more information on the LT and FRODOSpec check the following website http://telecope.livjm.ac.uk 